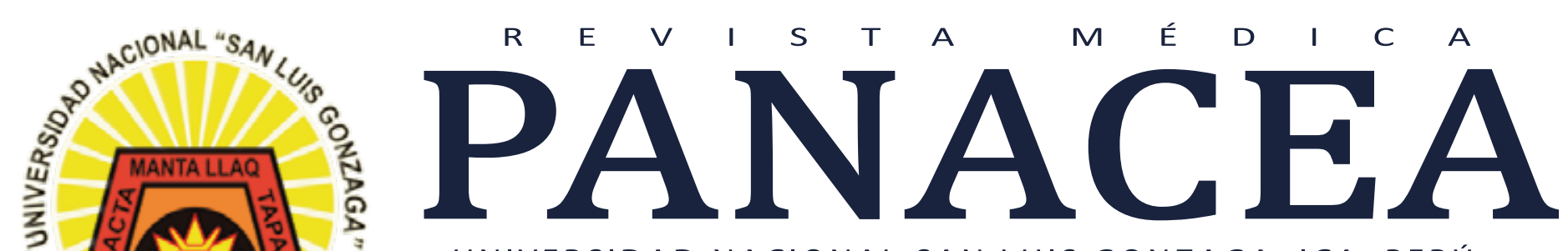

UNIVERSIDAD NACIONAL SAN LUIS GONZAGA. ICA, PERÚ

FACULTAD DE MEDICINA HUMANA "DANIEL ALCIDES CARRIÓN"

p-ISSN 2223-2893

e-ISSN 2225-6989

Volumen 9 Número 3 PUBLICACION CUATRIMESTRAL Setiembre - Diciembre 2020

ARTÍCULO DE REVISIÓN:

\title{
CARACTERÍSTICAS ULTRASONOGRÁFICAS DEL NERVIO MEDIANO EN PACIENTES CON SÍNDROME DEL TÚNEL CARPIANO.
}

ULTRASONOGRAPHIC CHARACTERISTICS OF THE MEDIAN NERVE IN PATIENTS WITH CARPAL TUNNEL SYNDROME.

\section{AUTORES:}

ATENAS ARTEAGA ROMANI

MELISA PAMELA QUISPE ILANZO

INDEXADA EN:

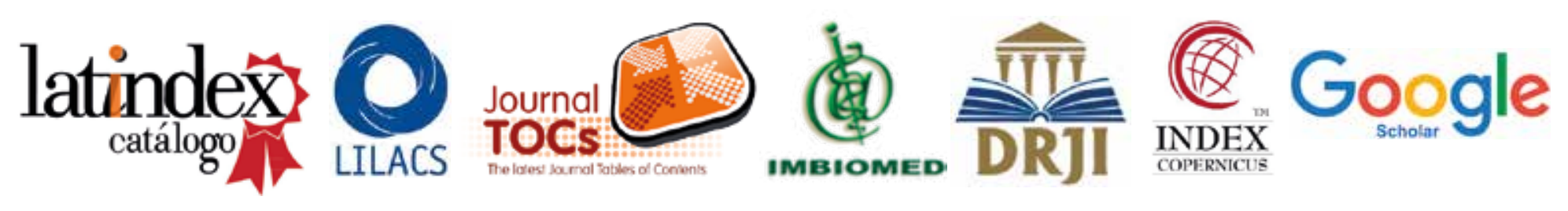

revistas.unica.edu.pe 


\section{CARACTERÍSTICAS ULTRASONOGRÁFICAS DEL NERVIO MEDIANO EN PACIENTES CON SÍNDROME DEL TÚNEL} CARPIANO.

Ultrasonographic characteristics of the median nerve in patients with carpal tunnel syndrome.

Atenas Arteaga-Romani ${ }^{1, a}$, Melisa Pamela Quispe-Ilanzo ${ }^{2, b}$.

1. Hospital Regional de Ica. Ica, Perú. https://orcid.org/0000-0002-3497-4781

2. Natural and Social Sciences Research. Ica, Perú. https://orcid.org/0000-0003-3695-591X

a. Médico Cirujano

b. Licenciada en Enfermería

DOI: https://doi.org/10.35563/rmp.v9i3.375

\section{Correspondencia:}

Dirección: Urb. Rinconada de

Huacachina Tercera etapa F- 09

Huacachina. Tercera etapa F- 09

Celular: 945557987

athe_14@hotmail.com

Contribuciones de autoría: - AAR y MPQI participaron en la concepción y diseño del manuscrito, recolección, análisis y explicación de los resultados, redacción y evaluación crítica del contenido del manuscrito y aprobación final del artículo.

Conflicto de intereses: no existen conflictos de intereses del autor o autores de orden económico, institucional laboral o personal.

Financiamiento:

Autofinanciado.

\section{Cómo citar:}

Arteaga-Romaní

Quispe-Ilanzo

C a r a c t erís tic a s ultrasonográficas del nervio mediano en pacientes con síndrome del túnel carpiano. Rev Méd Panacea. 2020;9(3) 194-197. DOl:

https://doi.org/10.35 563/rmp.v9i3.375

\section{RESUMEN}

Introducción: el síndrome del túnel del carpo es la patología más común e incapacitante de los miembros superiores. Objetivo: determinar las características ultrasonográficas del nervio mediano en el túnel del carpo en pacientes con síndrome del túnel carpiano. Materiales y métodos: se realizó una búsqueda sistemática de investigaciones publicadas entre los años 2014 y 2019, en las bases de datos especializadas en ciencias de la salud de acceso abierto: Google Académico, Ebsco, PubMed, Redalyc, Scielo y Lilacs. Resultados: a través de la búsqueda de artículos científicos se recopilaron cuatro artículos: tres internacionales y uno nacional. No se encontraron estudios locales. Conclusiones: Los estudios revisados, indican que los afectados por el síndrome del túnel carpiano -al examen ultrasonográfico- presentan medidas mayores del área de corte transversal del nervio mediano, siendo los hallazgos más evidentes: área seccional del nervio mediano de $16 \mathrm{~mm} 2$ (media de área seccional transversal del nervio mediano de 18,75mm2 a nivel de la entrada al túnel del carpo y 19,28mm2 en la salida). En las mujeres se presentó un área seccional de nervio mediano mayor en el agujero de entrada (19,77mm2) versus al varón (16,72mm2); asimismo, se visualizó un grosor del retináculo flexor hasta 1,82 mm y altura del túnel del carpo de 10,1 mm en todos los casos.

Palabras clave: túnel carpiano, síndrome, ecografía, ecográfico.

\section{ABSTRACT}

Introduction: carpal tunnel syndrome is the most common and disabling pathology of the upper limbs. Objective: to determine the ultrasonographic characteristics of the median nerve in the carpal tunnel in patients with carpal tunnel syndrome. Materials and methods: a systematic search was carried out for research published between 2014 and 2019, in the open access databases specialized in health sciences: Google Academic, Ebsco, PubMed, Redalyc, Scielo and Lilacs. Results: through the search for scientific articles, four articles were collected: three international and one national. No local studies were found. Conclusions: patients with carpal tunnel syndrome -on ultrasound examination- had bigger measurements of the cross-sectional area of the median nerve, the most common findings were: $16 \mathrm{~mm} 2$ for sectional area of the median nerve (mean of median nerve sectional area: $18.75 \mathrm{~mm} 2$ at the entrance to the carpal tunnel and $19.28 \mathrm{~mm} 2$ at the exit), females had a bigger sectional area of the median nerve at the entrance of foramen $(19.77 \mathrm{~mm} 2)$ when compared with males $(16.72 \mathrm{~mm} 2)$; likewise, it was observed that a bigger thickness of the flexor retinaculum up to $1.82 \mathrm{~mm}$ and a carpal tunnel height of $10.1 \mathrm{~mm}$ was present in all cases.

Keywords: carpal tunnel, syndrome, ultrasound, ultrasonographic. 


\section{INTRODUCCIÓN}

La mano y la muñeca son estructuras anatómicas frecuentemente comprometidas por una amplia variedad de enfermedades y síndromes. La mayor parte de dichas condiciones patológicas afectan a los tejidos blandos, y su precisa caracterización resulta particularmente compleja utilizando exclusivamente el examen físico(1).

El síndrome del túnel del carpo es la patología más común e incapacitante de los miembros superiores, generado por la compactación del nervio mediano en el túnel del carpo, manifestándose con dolor, parestesias y entumecimiento(1). Tiene una prevalencia del $3 \%$ al $5 \%$ en la población general y del $6 \%$ en el grupo de mujeres mayores de 40 años. Afecta más al sexo femenino, en su mayoría oficinistas(2,3), con índice de masa corporal mayor de 29; su distribución por edades es bimodal con una morbilidad máxima entre los 50 y los 59 años, y luego a partir de los 80 años a más. Puede ocurrir en una o ambas manos, aunque la manifestación bilateral es mucho más frecuente(2).

Se ha reportado que los síntomas bilaterales ocurren en el $22 \%$ $-87 \%$ de los pacientes. Sin embargo, la condición es más común en la mano dominante. La manifestación primaria o más intensa del síndrome bilateral -en un importante número de casos- se desarrolla en la mano dominante. En casi la mitad de los pacientes con síntomas unilaterales, se han encontrado anomalías de conducción en los estudios electrofisiológicos del nervio mediano contralateral a pesar de la ausencia de signos clínicos. En la mayoría de ellos, el síndrome se desarrolla meses o años después(2).

Por esta razón se realizó la presente búsqueda sistemática de artículos científicos, con el objetivo de determinar las características ultrasonográficas del nervio mediano en el túnel del carpo en pacientes con síndrome del túnel carpiano.

\section{MATERIALES Y MÉTODOS}

Se realizó una búsqueda sistemática de investigaciones publicadas entre los años 2014 y 2019, en las bases de datos de acceso abierto especializadas en ciencias de la salud: Google Académico, Ebsco, PubMed, Redalyc, Scielo y Lilacs, utilizando las palabras clave en español: "ULTRASONOGRAFIA", "ECOGRAFICA", "CARPO", "CARPIANO", "SINDROME" y "TUNEL"; y en inglés: "ULTRASOUND" "ULTRASONOGRAPHY", "ECOGRAPHY", "ECOGRAPHIC", "CARPUS", "CARPAL", "SYNDROME" and "TUNNEL". Se omitieron las secciones "Cartas al editor" y "Editorial"

\section{RESULTADOS}

A través de la búsqueda de artículos científicos se recopilaron cuatro artículos: tres internacionales y uno nacional. No se encontraron estudios locales.

Zamora (Guatemala, 2015)(4), en su estudio descriptivo "Ultrasonido de alta resolución en la evaluación de Facultad de Ciencias Médicas de la Universidad de San Carlos de Guatemala a pacientes con sospecha de síndrome del túnel del carpo", describe la función del ultrasonido de alta resolución en la evaluación de pacientes con sospecha de síndrome del túnel del carpo. El estudio se desarrolló con la revisión de 34 casos del departamento de traumatología (unidad de mano) del Hospital Roosevelt de los años 2011 al 2014. La intervención, empleada, fue la ecografía de alta resolución, en la muñeca sintomática, seguida de la cirugía de liberación del nervio mediano. De los casos, 22 fueron mujeres (64,7\%) y 12 varones (35,3\%); de ellos, el $100 \%$ reportó, al menos, una variación sugestiva en la patología hallada en el ultrasonido. Del ultrasonido los hallazgos más usuales, fueron: elevación del retináculo palmar $(94,11 \%)$ y pérdida del patrón fascicular (85,29\%); además, la media del área seccional transversal del nervio mediano fue de $18,75 \mathrm{~mm} 2$ a nivel de la entrada al túnel del carpo y 19,28mm2 en la salida. La mitad de los casos presentaron una variación del área seccional transversal mayor a $2 \mathrm{~mm} 2$ entre la entrada y salida del túnel carpiano; las pacientes del sexo femenino presentaron un área seccional de nervio mediano mayor en el agujero de entrada $(19,77 \mathrm{~mm} 2)$ versus el sexo masculino $(16,72 \mathrm{~mm} 2)$. Del intraoperatorio, los hallazgos más frecuentes fueron: aplanamiento del nervio mediano (100\%), engrosamiento del ligamento carpiano y edema y fibrosis perineural (94.11\%). El autor infiere que el mayor número de casos fueron mujeres; todos los casos presentaron hallazgos sugestivos de patología en el ultrasonido en muñeca sintomática y que en el intraoperatorio lo que más se encontró fue el aplanamiento del nervio mediano.

Rivas-Gallardo y col. (México, 2015)(5), en su estudio clínico transversal, observacional y descriptivo "Hallazgos ultrasonográficos más frecuentes en el síndrome de túnel del carpo" en el que se buscó la correlación entre la evaluación clínica y los resultados ultrasonográficos en la región del carpo con la finalidad de definir el diagnóstico de síndrome del túnel del carpo e identificar, posibles, causas estructurales o anatómicas concomitantes que condicionan la compresión extrínseca del nervio mediano. Se analizaron datos de 25 pacientes con diagnóstico de síndrome del túnel del carpo por estudio de electromiografía del Hospital General de México. De ellos, el $84 \%$ de los pacientes eran mujeres y $16 \%$ hombres, cuyas edades oscilaban entre los 29 a 64 años y que la gran mayoría realizaban actividades que involucran el uso repetitivo de la muñeca y de la mano. Los tres hallazgos sonográficos más repetitivos, debidos al síndrome del túnel del carpo, fueron: área seccional del nervio mediano de $16 \mathrm{~mm} 2$, hasta $1,82 \mathrm{~mm}$ de grosor del retináculo flexor y con una altura del túnel del carpo de 10,1 mm. Los autores concluyen que el síndrome del túnel del carpo es la neuropatía compresiva más frecuente de la extremidad superior; además, se relacionó el tipo de actividad y la presencia de la afección directa en la mano dominante. Asimismo las mediciones ultrasonográficas descritas son similares a las descritas en la literatura internacional especializada y es posible visualizar estructuras anatómicas vasculares, musculares y óseas regionales.

Bueno-Gracia y col. (España, 2015)(6), en el estudio observacional "Validez diagnóstica de la ecografía en el síndrome del túnel carpiano", en él se buscó definir la validez diagnóstica de las mediciones ecográficas del área de sección transversal del nervio mediano en la muñeca (AST-M) y el ratio del área del nervio mediano entre la muñeca y el antebrazo (R-MA) para el diagnóstico del STC, empleando el electroneurograma. Se evaluaron a 59 participantes (97 muñecas), los cuales fueron referidos para someterse a un electroneurograma por sospecha de síndrome del túnel carpiano. Los resultados indicaron que, con un punto de corte 
de $9,15 \mathrm{~mm} 2$, la medición AST-M obtuvo una sensibilidad del $75,81 \%$ y una especificidad del $74,29 \%$; en el ratio de probabilidad positiva fue de 2,95 y una ratio de probabilidad negativa de 0,33 . Para la medición R-MA y un punto de corte de 1,56, los valores de sensibilidad, especificidad y ratios de probabilidad positiva y negativa fueron $70,97 \%, 71,43 \%, 2,48$ y 0,4 , respectivamente. Los autores afirman que tanto el AST-M como la R-MA son medidas útiles en el diagnóstico del síndrome del túnel carpiano tomando como prueba de referencia el electroneurograma.

Sandoval (Perú, 2016)(7), en el estudio observacional, analítico y transversal "Asociación clínica-ultrasonografía en el diagnóstico del síndrome de túnel carpiano" realizado con el objetivo de determinar la asociación clínico - ultrasonográfico para el diagnóstico del síndrome de túnel carpiano en el Centro de Especialidades Florencia de Mora-UPAO. En el estudio conto con 35 pacientes con sospecha de síndrome de túnel carpiano, donde se midió, por ultrasonografía, el área del corte transversal del nervio mediano en la entrada del túnel carpiano de ambos carpos y se examinó la presencia de los criterios clínicos considerados. Del total de participantes, 28 fueron mujeres (80\%) y 7 varones (20\%), la media de edad fue de 49,26 \pm 9,13 años; además, 7 de los participantes tuvieron criterios clínicos compatibles de forma bilateral y 24 de forma unilateral, siendo la mano dominante la más afectada. En el estudio se calculó el coeficiente de correlación de Sperman " $r$ ", encontrándose un valor de $r=0.94$ y $p<0.001$; así mismo, se aplicó la prueba de t de Student encontrándose la media del área de corte transversal del nervio mediano de los pacientes con síndrome del túnel carpiano fue mayor, con un valor de $p<0,000$. El autor deduce que existe una asociación entre el área de corte transversal del nervio mediano y la aparición de síntomas clínicos de síndrome del túnel carpiano además que, los pacientes con síndrome del túnel carpiano presentan medidas mayores en el área de corte transversal del nervio mediano.

\section{DISCUSIÓN}

El síndrome del túnel carpiano suele describirse como una neuropatía por compresión que afecta al nervio mediano a nivel del ligamento carpiano transverso. Se ha relacionado con vibraciones, movimientos de dedos, manos y muñecas muy repetitivos y enérgicos $(2,8)$.

Actualmente, el diagnóstico de lesión nerviosa periférica se basa en la valoración clínica y electrodiagnóstico. Los estudios de electrodiagnóstico incluyen estudios de conducción nerviosa y electromiografía. Los estudios de conducción nerviosa confirman el síndrome del túnel carpiano al detectar una alteración de la conducción del nervio mediano a través del túnel carpiano, pero con conducción normal en otros lugares. La electromiografía evalúa los cambios patológicos en los músculos inervados por el nervio mediano, típicamente el músculo abductor del pulgar corto(9). Sin embargo, el ultrasonido es una herramienta valiosa y de bajo costo que permite caracterizar la fibra neural y sus componentes(10), con sensibilidad y especificidad similares a las de los estudios de electrodiagnóstico(11), pero requiere de un gran conocimiento de los detalles anatómicos y de la estrecha correlación entre los hallazgos imagenológicos con la clínica del paciente, así como también con los resultados de los estudios electrofisiológicos(12).
Los equipos ultrasonográficos permiten una visualización clara de estructuras anatómicas con una resolución espacial incluso inferior a $1 \mathrm{~mm}(1)$. Gracias a los equipos de alta frecuencia y tecnología compacta su uso es más frecuente en la valoración de los nervios periféricos, permitiendo la identificación de los componentes anatómicos sutiles de igual o mejor manera que la resonancia magnética. A su vez, esta tecnología, permite la identificación de un amplio gama de estados anómalos que afectan a los nervios $(1,12)$.

Además, los equipos ultrasonográfica demuestran múltiples ventajas sobre la resonancia magnética, tales como: mejor resolución espacial, posibilidad de explorar largos tramos de los troncos nerviosos en un único estudio; estudiar de forma comparativa con el mapa anatómico contralateral; y examinar tanto de manera estática o dinámica en tiempo real(12). En el síndrome del túnel carpiano permite identificar las diversas variaciones morfológicas que se verifican a nivel del nervio mediano pro-piamente dicho $\mathrm{y} / \mathrm{o}$ de las estructuras vecinas(13).

Después de la presente revisión, se puede concluir que, los pacientes afectados por el síndrome del túnel carpiano -al examen ultrasonográfico- presentan dimensiones mayores del área de corte transversal del nervio mediano, demostrado en: área seccional del nervio mediano de $16 \mathrm{~mm} 2$ (media de área seccional transversal del nervio mediano de $18,75 \mathrm{~mm} 2$ a nivel de la entrada al túnel del carpo y 19,28 mm2 en la salida). Además, al parecer el sexo femenino, afectado por el síndrome, presenta un área seccional de nervio mediano mayor en el agujero de entrada $(19,77 \mathrm{~mm} 2)$ en comparación el sexo masculino (16,72 mm2); asimismo, se observó mayor grosor del retináculo flexor de hasta $1,82 \mathrm{~mm}$ y altura del túnel del carpo de 10,1 $\mathrm{mm}$ en todos los casos. 


\section{REFERENCIAS BIBLIOGRÁFICAS}

1. De la Paz M. Indicadores ecográficos de severidad clínica en pacientes con síndrome de túnel del carpo unilateral atendidos en la clínica de neurofisiología del Hospital Bautista en el período de agosto 2014 a enero 2015. Managua: Facultad de Ciencias Médicas de la Universidad Nacional Autónoma de Nicaragua. 2015.

2. Dec $P$, Zyluk A. Bilateral carpal tunnel syndrome $-\mathrm{A}$ review. Polish Journal of Neurology and Neurosurgery. 2018; 52:79-83.

3. Guerrón DC, Guevara GM. Características clínicas, manejo y evolución de pacientes con síndrome de túnel carpiano. Hospital José Carrasco Arteaga, enero 2013 - diciembre 2016. Ecuador: Facultad de Ciencias Médicas de la Universidad de Cuenca. 2017.

4. Zamora EA. Ultrasonido de alta resolución en la evaluación d Facultad de Ciencias Médicas de la Universidad de San Carlos de Guatemalae pacientes con sospecha de síndrome del túnel del carpo. Guatemala: Facultad de Ciencias Médicas de la Universidad de San Carlos de Guatemala. 2016

5. Rivas-Gallardo BP, Guerrero-Avendaño G, González-de la Cruz J. Hallazgos ultrasonográficos más frecuentes en el síndrome de túnel del carpo. Anales de Radiología México. 2015; 14(4):371-376.

6. Bueno-Gracia E, Haddad-Garay M, Tricás-Moreno JM, Fanlo-Mazas

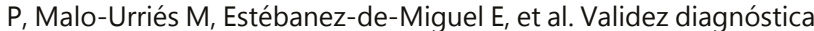
de la ecografía en el síndrome del túnel carpiano. Rev Neurol 2015; 61:1-6.

7. Sandoval FP. Asociación clínica-ultrasonográfica en el diagnóstico del síndrome de túnel carpiano. La Libertad: Facultad de Medicina Humana de la Universidad Privada Antenor Orrego. 2016.

8. Balbastre M, Andani J, Garrido R, López A. Análisis de factores de riesgo laborales y no laborales en Síndrome de Túnel Carpiano (STC) mediante análisis bivariante y multivariante. Rev Asoc Esp Espec Med Trab. 2016; 25(3):126-141.

9. Wipperman J, Goerl K. Carpal Tunnel Syndrome: Diagnosis and Management. American Family Physician. 2016; 94(12):993-999.

10. Hofmann-González F, Hernández-Díaz C. Evaluación del sistema nervioso periférico por ultrasonido. Rev. chil. reumatol. 2014; 30(4):153-160.

11. Bland JD, Rudolfer SM. Ultrasound imaging of the median nerve as a prognostic factor for carpal tunnel decompression. Muscle Nerve 2014; 49(5):741-744.

12. Arruti A. Ultrasonografía en las neuropatías periféricas frecuentes del miembro superior. Ensayo iconográfico. Rev. Imagenol. 2017 21(1):18-34

13. Benguria-Arrate G, Gutiérrez-Ibarluzea I, Bayón Yusta JC, Galnares-Cordero L. Informes de evaluación de tecnologías sanitarias: Indicaciones, utilidad y uso de la ecografía en Atención Primaria. España: Servicio Central de Publicaciones del Gobierno Vasco. 2019.

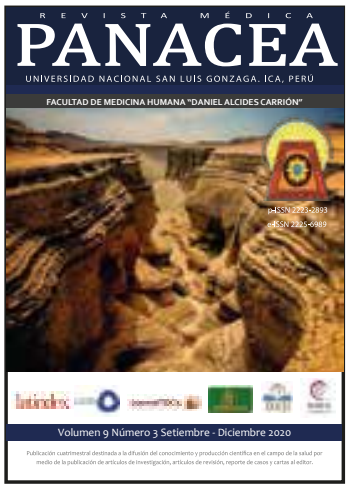

